\title{
Persistence and adherence to interferon and glatiramer acetate in patients with multiple sclerosis
}

\author{
Emma Bartolomé-García, Ángela Usarralde-Pérez, Patricia Sanmartín-Fenollera, \\ Monserrat Pérez-Encinas
}

Department of Pharmacy, Hospital Universitario Fundación Alcorcón, Alcorcón Madrid, Spain

\section{Correspondence to} Dr Emma Bartolomé-García, Departmentof Pharmacy, Hospital Universitario Fundación Alcorcón, Alcorcon, Madrid,Spain; emmabgv@ gmail.com

Received 12 April 2017 Revised 25 July 2017 Accepted 8 August 2017 Published Online First 31 August 2017

EAHP Statement 4: Clinical Pharmacy Services. EAHP Statement 5: Patien Safety and Quality Assurance.
D) Check for updates

To cite: Bartolomé-García $\mathrm{E}$ Usarralde-Pérez Á,

Sanmartín-Fenollera P, et al. Eur J Hosp Pharm

2019:26:23-28.

\begin{abstract}
Objectives To analyse persistence and adherence in patients with multiple sclerosis receiving first-line treatment with subcutaneous glatiramer acetate $20 \mathrm{mg}(\mathrm{GA})$, subcutaneous interferon $\beta 1 \mathrm{a}$ (IFN $\beta 1 \mathrm{a}$ sc), intramuscular interferon $\beta 1$ a (IFN $\beta 1$ a-im) and subcutaneous interferon $\beta 1 \mathrm{~b}$ (IFN $\beta 1 \mathrm{~b}$-sc) and to identify associated factors and reasons for discontinuation.
\end{abstract} Methods An observational retrospective study was performed between January 1999 and November 2014. Persistence was defined as the time from treatment initiation until discontinuation, and adherence as the number of units dispensed since treatment initiation until its interruption divided by the theoretical number of units needed to cover said period as a percentage. A patient was considered adherent if $\geq 95 \%$. Persistence was measured using the Kaplan-Meier method and univariate Cox regression; adherence was measured using a univariate binary logistical regression model. Results The study included 224 patients. The median persistence was 1349 days (95\% Cl 1017.4 to 1680.6). Patients receiving IFN $\beta 1$ a-im continued treatment for a longer time (1720 days; 95\% Cl 1196.8 to 2243.2), while patients treated with IFN $\beta 1$ a-sc had the lowest persistence (771 days; $95 \% \mathrm{Cl} 377.4$ to 1164.6 ) ( $H R=1.7 ; 95 \% \mathrm{Cl} 1.02$ to 2.72). Patients with Expanded Disability Status Scale (EDSS) 1.5-6 discontinued treatment earlier than those with EDSS 0-1 (HR 1.5; $95 \%$ Cl 1.01 to 2.25$) ; 94.4 \%$ of patients discontinued treatment due to medical decision, primarily due to lack of efficacy (24.6\%) and adverse effects (17.4\%), while $80.8 \%$ of patients had good adherence. GA had the highest adherence, with no major difference from IFN $\beta 1$ a-im, while IFN $\beta 1 \mathrm{~b}$-sc showed the highest nonadherence (OR 3.5; 95\% Cl 1.29 to 9.28).

Conclusions The persistence levels obtained were lower than in similar studies. EDSS was identified as an independent predictor of treatment interruption. Acceptable adherence was achieved among the population, comparable to other studies and influenced by the drug.

\section{INTRODUCTION}

Multiple sclerosis (MS) is a progressive chronic inflammatory disease of the central nervous system with autoimmune causes, characterised by the presence of multiple demyelinating plaques spread throughout the brain and the spinal cord. There has been an increase in the incidence and prevalence of the disease, but this has not been caused by better diagnostic methods that allow an earlier diagnosis but, rather, it seems to be caused by environmental factors. ${ }^{1}$ Recent epidemiological studies have classified Mediterranean Europe as a high-risk region; in Spain the mean prevalence is 100 patients per 100000 inhabitants. ${ }^{1}$

MS symptomatology can be very diverse, depending on the type of disease, location of lesions and evolution stage. There are various forms of evolution according to the classification by Lublin et al: relapsing-remitting MS (RRMS), primary progressive MS (PPMS), secondary progressive MS (SPMS) and progressive-relapsing MS (PRMS). RRMS affects $85 \%$ of patients and presents with flare-ups; PPMS affects $10 \%$ of patients, who have no defined flare-ups but constant worsening; SPMS is the form of the disease to which $30-50 \%$ of patients with RRMS will progress and is characterised by continuous progression; and PRMS is very infrequent and has a severe prognosis. ${ }^{2}$ The clinically isolated syndrome (CIS), which is the first clinical presentation of the disease, has recently been described, while the radiologically isolated syndrome (RIS) describes those cases where incidental imaging findings suggest inflammatory demyelinating lesions in the absence of clinical signs or symptoms. ${ }^{2}$

Currently, only disease-modifying therapies (DMTs) are available, which reduce the number of flare-ups and slow disease progression. The current treatment pattern is a sequential monotherapy, starting with treatments with moderate efficacy but high safety and continuing with drugs which are more potent but present a more adverse toxicity profile-which is why we speak, conventionally, about first- and second-line drugs. ${ }^{3}$ MS presents characteristics of a chronic disease and therefore factors such as adherence and persistence will have an impact on treatment efficacy. ${ }^{4}$

Adherence is defined by the International Society for Pharmacoeconomics and Outcomes Research as the extent to which a patient acts in accordance with the prescribed interval and dose and dosing regime. ${ }^{5}$ Equally, the World Health Organization defines adherence as the extent to which the patient's history of therapeutic drug-taking coincides with the treatment prescribed by the healthcare professional. ${ }^{6}$ Persistence is defined as the time during which the patient continues treatment-that is, the time elapsed from initiation until interruption. ${ }^{5}$ The measurement of persistence is relevant, among other reasons, because a minimum time of treatment duration is required for drugs to be effective.

Similar to other chronic conditions, the analysis of adherence and persistence in MS becomes relevant due to the correlation between a chronic 
condition and a reduction in their rates. ${ }^{6}$ Different studies on DMTs have been conducted, which show the existence of multiple factors that can have an influence on adherence and persistence including patients getting tired of chronic treatment, perceived loss of efficacy, adverse effects, problems with the injection device and dosing frequency. ${ }^{78} \mathrm{~A}$ recent Canadian study showed aspects associated with non-adherence such as loss of cognitive ability, disease duration, Expanded Disability Status Scale (EDSS) and alcohol dependency. ${ }^{9}$ Regarding persistence, Correia et al identified baseline EDSS as a discontinuation factor. ${ }^{10}$

The primary objective of our study is an overall analysis of persistence and adherence among DMTs with parenteral subcutaneous and intramuscular administration (DMTps) as first-line treatment for patients with a diagnosis of MS. Our secondary objective is to identify the associated factors and reasons for discontinuation.

\section{METHODS}

An observational retrospective study was performed in naïve patients with a diagnosis of MS who started first-line treatment in a University Hospital between 1 January 1999 and 30 November 2014. The follow-up period was from treatment initiation until discontinuation or the end of the study (May 2015). Patients who started treatment in another centre were excluded.

The parenteral DMTs analysed were those marketed at the time of treatment initiation: subcutaneous glatiramer acetate $20 \mathrm{mg}$ (GA), subcutaneous interferon $\beta 1 \mathrm{a}$ (IFN $\beta 1 \mathrm{a}-\mathrm{sc})$, intramuscular interferon $\beta 1 \mathrm{a}$ (IFN $\beta$ 1a-im) and subcutaneous interferon $\beta 1 \mathrm{~b}$ (IFN $\beta 1 \mathrm{~b}-\mathrm{sc}$ ).

Patients were selected through the Pharmacy application for Outpatients, and clinical data were obtained from the review of electronic clinical records as well as from the forms requesting treatment initiation. The variables collected were: gender, date of birth, form of evolution, EDSS before treatment initiation, firstline drug, dates of treatment initiation and interruption, units dispensed and reasons for interruption. Treatment interruption was classified depending on who took the decision-the neurologist or the patient.

Persistence was defined as the time in days from treatment initiation until discontinuation for any cause, ${ }^{7}$ according to Pharmacy records. For periods between dispensing $>1.5$ times the time covered by the medication dispensed, persistence was confirmed through the electronic clinical record. Patients do not need to go for refills with a new prescription each time; prescriptions are valid until the next doctor's appointment in several months' time. The sequencing of the different doses of IFN $\beta 1$ a-sc was not considered discontinuation. Treatment interruption refers to the percentage of treatment interruption at any time of follow-up. The adherence rate (A) was calculated as the number of units dispensed since treatment initiation and until discontinuation (D) divided by the theoretical number of units needed to cover said period $(N) \times 100$ times $(A=(D / N) \times 100)$. A patient was considered adherent if $\geq 95 \%$. ${ }^{11}$ For the mean calculation, adherence $>100 \%$ (data resulting from medication collection in advance) was considered as $100 \%$ adherence.

\section{Analysis of data}

A descriptive analysis was conducted, presenting categorical baseline variables as absolute frequencies and percentages and continuous variables as means and SD. Comparison of the baseline demographic characteristics (gender, age, form of evolution and EDSS) between the treatment arms was conducted using the ANOVA test, $\chi^{2}$ test or Fisher exact test. For analysis, patients were classified into two groups according to mean age and EDSS in order to obtain two arms of similar size. Differences in persistence were estimated based on the drug and demographical variables using Kaplan-Meier survival techniques (log-rank test) and univariate Cox regression. The analysis of adherence by parenteral DMTs and the determination of the association between adherence and the different demographical variables was analysed using a univariate binary logistical regression model; the group with the highest adherence was used as a

\begin{tabular}{|c|c|c|c|c|c|c|}
\hline & $\mathrm{GA}(\mathrm{n}=54)$ & IFNß1a-sc $(n=47)$ & IFNß1a-im (n=73) & IFN $\beta 1 b-s c(n=50)$ & Overall $(n=224)$ & $p$ Value \\
\hline \multicolumn{7}{|l|}{ Gender, n(\%) } \\
\hline Male & $17(31.5)$ & $20(42.6)$ & $21(28.8)$ & $20(40.0)$ & $78(34.8)$ & $0.35^{*}$ \\
\hline Female & $37(68.5)$ & $27(57.4)$ & $52(71.2)$ & $30(60.0)$ & $146(65.2)$ & \\
\hline \multicolumn{7}{|l|}{ Age (years) } \\
\hline Mean & 36.2 & 35.8 & 36.7 & 36.9 & 36.4 & $0.96 t$ \\
\hline SD & 8.7 & 10.4 & 10.4 & 11.5 & 10.2 & \\
\hline \multicolumn{7}{|c|}{ Groups, n (\%) } \\
\hline$\leq 36$ years & $27(50)$ & $25(53.2)$ & $36(49.3)$ & $28(56)$ & $116(51.8)$ & $0.89^{*}$ \\
\hline$>36$ years & $27(50)$ & $22(46.8)$ & $37(50.7)$ & $22(44)$ & $108(48.2)$ & \\
\hline \multicolumn{7}{|c|}{ Evolution forms, n (\%) } \\
\hline RRMS & $54(100.0)$ & $41(87.2)$ & $70(95.9)$ & $41(82.0)$ & $206(92)$ & $0.00 \ddagger$ \\
\hline SPMS & $0(0.0)$ & $5(10.7)$ & $3(4.1)$ & $6(12.0)$ & $14(6.2)$ & \\
\hline PPMS & $0(0.0)$ & $1(2.1)$ & $0(0.0)$ & $3(6.0)$ & $4(1.8)$ & \\
\hline \multicolumn{7}{|c|}{ EDSS, $n(\%)(n=186) \S$} \\
\hline 0-1 & $27(52.9)$ & $13(43.3)$ & $32(51.6)$ & $14(32.6)$ & $86(46.2)$ & $0.17^{*}$ \\
\hline $1.5-6$ & $24(47.1)$ & $17(56.7)$ & $30(48.4)$ & $29(67.4)$ & $100(53.8)$ & \\
\hline
\end{tabular}

\footnotetext{
*Pearson's $\chi^{2}$ test.
}

tOne-way ANOVA test.

\#Fisher's exact test.

§EDSS data were unknown for 38 patients.

EDSS, Expanded Disability Status Scale; GA, glatiramer acetate; IFN $\beta$ 1a-im, intramuscular interferon $\beta 1$; IFN $\beta 1$ a-sc, subcutaneous interferon $\beta 1$; IFN $\beta 1$ b-sc, subcutaneous interferon $\beta 1$; PPMS, primary progressive multiple sclerosis; RRMS, relapse-remitting multiple sclerosis; SPMS, secondary progressive multiple sclerosis. 
Table 2 Analysis of persistence by Kaplan-Meier and Cox regression (univariate analysis)

\begin{tabular}{|c|c|c|c|c|c|}
\hline & $\mathrm{GA}(\mathrm{n}=54)$ & IFNß1a-sc $(n=47)$ & IFNß1a-im ( $n=73)$ & IFN $\beta 1 b-s c(n=50)$ & Overall $(n=224)$ \\
\hline Median, days & 1381 & 771 & 1720 & 992 & 1349 \\
\hline $95 \% \mathrm{Cl}$ & 413.8 to 2348.2 & 377.4 to 1164.6 & 1196.8 to 2243.2 & 84.6 to 1899.4 & 1017.4 to 1680.6 \\
\hline Interruption percentage (\%) & 51.8 & 74.5 & 41.1 & 64 & 55.8 \\
\hline \multicolumn{6}{|l|}{ Probability of persistence (\%) } \\
\hline 6 months & 88.7 & 91.5 & 95.9 & 90 & 91.9 \\
\hline 7 years & 12.2 & 21.5 & 28.1 & 22.9 & 23.1 \\
\hline HR & 1.4 & 1.7 & Reference & 1.6 & \\
\hline $95 \% \mathrm{Cl}$ & 0.85 to 2.38 & 1.02 to 2.72 & & 1.00 to 2.68 & \\
\hline p Value & 0.18 & 0.04 & & 0.06 & \\
\hline
\end{tabular}

GA, glatiramer acetate; IFN $\beta 1$ a-im, intramuscular interferon $\beta 1$ a; IFN $\beta 1$ a-sc, subcutaneous interferon $\beta 1$; IFN $\beta 1$ b-sc, subcutaneous interferon $\beta 1 b$.

reference for each variable. The statistical program SPSS V.19.0 was used for data analysis.

Confidentiality regarding personal details was maintained according to the current national legislation.

\section{RESULTS}

The study included 244 patients. Their mean (SD) age at treatment initiation was 36.4 (10.2) years and $65.2 \%$ were female. The predominant form of MS was RRMS (92\%). Regarding treatment, $32.6 \%$ of patients received IFN $\beta 1 \mathrm{a}-\mathrm{im}, 24.1 \%$ were treated with GA, 22.3\% with IFN $\beta 1$ b-sc and 21\% with IFN $\beta 1 \mathrm{a}$-sc. Table 1 shows the demographic and clinical baseline characteristics of the population. The four treatment arms were homogeneous with regard to gender, age and EDSS, but not in terms of form of disease evolution. All patients in the GA arm had RRMS while around 10\% of patients in the IFN $\beta 1 \mathrm{a}$-sc and IFN $\beta 1$ b-sc arms had SPMS. Approximately half of the population with EDSS on record had values between 0 and 1 and none of them had EDSS $\geq 6.5$.

By the end of the study, $55.8 \%$ of patients had discontinued treatment, $36.6 \%$ were still on treatment and $7.6 \%$ of patients were lost to follow-up.

The median time of overall persistence estimated using the Kaplan-Meier method was 1349 days (95\% CI 1017.4 to

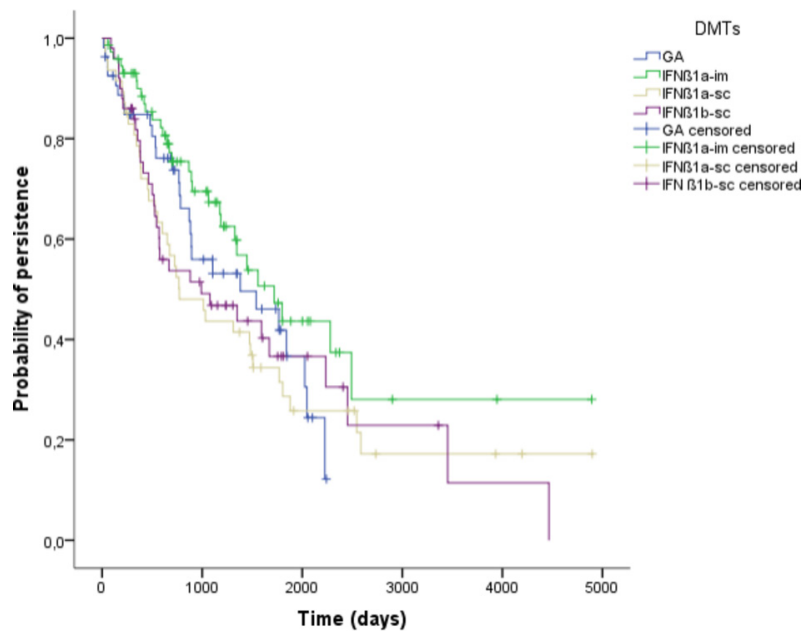

Figure 1 Kaplan-Meier survival curves (persistence) for glatiramer acetate (GA), intramuscular interferon $\beta 1$ a (IFN $\beta$ 1a-im), subcutaneous interferon $\beta 1$ a (IFN $\beta 1 \mathrm{a}-\mathrm{sc}$ ) and subcutaneous interferon $\beta 1 \mathrm{~b}$ (IFN $\beta 1 \mathrm{~b}$-sc).
1680.6). The probabilities of persistence at 6 months and 1,3 and 7 years were $91.9 \%, 83.9 \%, 54.6 \%$ and $23.1 \%$, respectively. There were no statistical differences in time of treatment continuation between drugs $(p=0.16)$. However, it was observed that patients receiving IFN $\beta 1 \mathrm{a}$-im continued treatment for a longer time. Taking IFN $\beta 1 \mathrm{a}$-im as a reference, there was a statistical difference in persistence for IFN $\beta 1$ a-sc with HR 1.7 (95\% CI 1.02 to 2.72 ) (see table 2 and figure 1 ).

The reason for discontinuation was a clinical decision in $94.4 \%$ of cases, mostly due to lack of efficacy $(24.6 \%)$ and adverse effects (17.4\%) (figure 2). Among those patients who did not continue treatment beyond 6 months, treatment discontinuation was due to toxicity in $82.4 \%$ of cases.

Regarding the study on adherence, $80.8 \%$ of patients had good adherence with a mean rate of $97.2 \%$. Adherence was highest with GA and was not significantly different from IFN $\beta 1 a-i m$, while IFN $\beta 1 \mathrm{~b}$-sc had the highest non-adherence rate with a risk 3.5 times higher than that of GA (table 3).

In the overall study on persistence and adherence by gender, age, form of evolution and EDSS, we did not find any significant differences except regarding persistence for EDSS. The risk of discontinuation estimated for patients with EDSS 1.5-6 was 1.51 times higher than that for patients with EDSS 0-1 (table 4).

Treatment discontinuation occurred in 58\% of adherent patients and in $46.5 \%$ of non-adherent patients $(p=0.17)$. After analysing adherence data according to the reasons for treatment interruption, $13.1 \%$ of patients who discontinued due to lack of efficacy were non-adherent, as well as $13.3 \%$ of patients who interrupted treatment due to adverse effects. Seven patients interrupted treatment voluntarily and three of them were non-adherent.

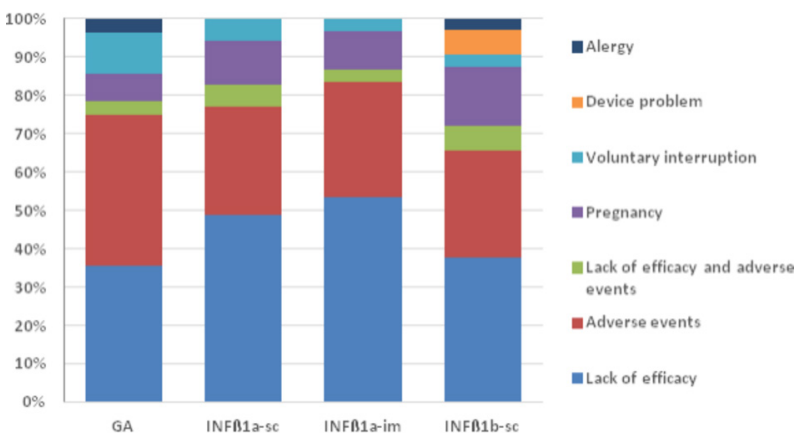

Figure 2 Reasons for treatment discontinuation. 
Original article

Table 3 Analysis of adherence through univariate binary logistical regression

\begin{tabular}{|c|c|c|c|c|c|}
\hline & $\mathrm{GA}(\mathrm{n}=54)$ & IFNB1a-sc $(n=47)$ & IFNß1a-im (n=73) & IFNB1b-sc $(n=50)$ & Overall $(n=224)$ \\
\hline Adherence, ${ }^{*} \mathrm{n}(\%)$ & $47(87.0)$ & $38(80.9)$ & $63(86.3)$ & $33(66.0)$ & $181(80.8)$ \\
\hline ORt & - & 1.6 & 1.1 & 3.5 & \\
\hline $95 \% \mathrm{Cl}$ & & 0.54 to 4.67 & 0.38 to 3.01 & 1.29 to 9.28 & \\
\hline p Value & & 0.40 & 0.90 & 0.01 & \\
\hline
\end{tabular}

*Percentage of patients with adherence $\geq 95 \%$.

tRisk of non-adherence vs GA.

GA, glatiramer acetate; IFN $\beta$ 1a-im, intramuscular interferon $\beta 1$ a; IFN $\beta 1$ a-sc, subcutaneous interferon $\beta 1 \mathrm{a}$; IFN $\beta 1 \mathrm{~b}$-sc, subcutaneous interferon $\beta 1 \mathrm{~b}$.

\section{DISCUSSION}

The benefits of continuous treatment in the setting of chronic conditions have been well documented; in the case of MS, these translate into a slower progression of the disease. Previous studies have shown that those patients with higher adherence and persistence have a lower risk of relapse and a better quality of life; this is also associated with a lower use of healthcare resources. ${ }^{71213}$ For these reasons, it is relevant to study treatment persistence and adherence, such as has been analysed in this retrospective study on naïve patients who have started firstline treatment with parenteral DMTs over a 16-year period.

The literature on persistence and adherence studies shows a lack of homogeneity in the measurement methods and definitions, as pointed out by other authors. ${ }^{14}$ Thus, we have observed variability when comparing persistence results with other studies, possibly due to differences in design, sample size and definitions. The retrospective series by Correia et al ${ }^{10}$ shows similarities to our study and our discontinuation rate, although the median persistence in our study was lower (1349 days vs 1614 days). Both studies had similar definitions of persistence and both were retrospective single-centre studies. The differences between them may therefore be due to the fact that, in the Portuguese study, the patients presented exclusively with the RRMS and CIS forms of the disease. In addition, the outcomes could have been affected by variability in the clinical management of patients, a reflection of the existing lack of consensus on criteria for progression to treatment, a factor that we have not analysed. Our estimations for persistence at 6 months and 1 year are higher than those obtained in studies conducted with large healthcare system databases such as the cohort studies by Evans et $a l^{15}$ and Reynolds et $a l .{ }^{16}$ Sample size is probably a decisive factor for these differences.

No significant differences in terms of persistence were found between drugs, although patients on IFN $\beta 1 \mathrm{a}$-im had a higher persistence rate, followed by GA with similar values and IFN $\beta 1$ a-sc in last place (in our study we did not differentiate the two dosing regimens for IFN $\beta 1 \mathrm{a}-\mathrm{sc}$ ). We observed high persistence values with IFN-sc at 6 months, possibly due to dose escalation at treatment initiation. These outcomes are in agreement with the observations by Reynolds et $a l^{16}$ and Correia et $a l{ }^{10}$ The values obtained for GA are very similar to those for IFN $\beta 1 \mathrm{a}$-im, so we cannot associate frequency of administration with persistence, as suggested by Correia et al. ${ }^{10}$

According to the literature available, we found a higher tendency for interruption of treatment in women and in younger patients. ${ }^{16}$ We also observed that EDSS had a significant influence on persistence. Patients with more advanced disability will discontinue treatment earlier; the explanation put forward by Correia $e t ~ a l^{10}$ is the need to change lines of treatment in order to prevent future progressions, which is a hypothesis not analysed in our study. It was not possible to assess the association between persistence and the form of disease evolution due to lack of homogeneity between the groups.

In the majority of cases the decision to interrupt treatment was made by the neurologist, mostly due to lack of efficacy and adverse effects; this agrees with findings in the literature in both retrospective ${ }^{10}$ and prospective series. ${ }^{17}$ When analysing each drug, IFN $\beta 1 \mathrm{a}$-im had the highest rate of discontinuation due to lack of efficacy, which agrees with the findings of other authors. ${ }^{10}$ GA had the highest rate of interruption due to adverse

Table 4 Analysis of persistence and adherence according to demographical variables

\begin{tabular}{|c|c|c|c|c|}
\hline & \multicolumn{2}{|c|}{ Persistence } & \multicolumn{2}{|c|}{ Adherence } \\
\hline & HR & $95 \% \mathrm{Cl}$ ( $\mathrm{p}$ value) & OR & $95 \% \mathrm{Cl}$ ( $\mathrm{p}$ value) \\
\hline \multicolumn{5}{|l|}{ Gender } \\
\hline Male & - & - & - & - \\
\hline Female & 1.3 & 0.91 to $1.94(0.14)$ & 1.3 & 0.65 to $2.55(0.74)$ \\
\hline \multicolumn{5}{|l|}{ Age } \\
\hline$\leq 36$ years & 1.2 & 0.82 to $1.67(0.38)$ & 1.2 & 0.60 to $2.25(0.67)$ \\
\hline$>36$ years & - & - & - & - \\
\hline \multicolumn{5}{|c|}{ Form of evolution } \\
\hline RRMS & - & - & 4.6 & 0.62 to $33.48(0.14)$ \\
\hline PPMS & 1.7 & 0.93 to $2.98(0.09)$ & - & - \\
\hline SPMS & 2.5 & 0.61 to $10.29(0.20)$ & 2.5 & 0.26 to $24.38(0.43)$ \\
\hline \multicolumn{5}{|l|}{ EDSS } \\
\hline 0-1 & - & - & 1.1 & 0.48 to $2.37(0.87)$ \\
\hline $1.5-6$ & 1.51 & 1.01 to $2.25(0.04)$ & - & - \\
\hline
\end{tabular}

EDSS, Expanded Disability Status Scale; GA, glatiramer acetate; PPMS, primary progressive multiple sclerosis; RRMS, relapse-remitting multiple sclerosis; SPMS, secondary progressive multiple sclerosis. 
effects, possibly because of its daily administration and cutaneous toxicity. This relationship has not been found in other studies ${ }^{10}$ and contrasts with the view that GA is better tolerated than interferons. ${ }^{18}$

It is estimated that half of patients with chronic diseases have low treatment adherence, ${ }^{19}$ which is why we highlight the acceptable adherence rate in our population (80.8\%). It is difficult to compare adherence rate results because different studies will use different definitions and different methods of calculation. Following a review of the literature, we consider that our study was reasonably demanding ( $\geq 95 \%)$. Thus, the Global Adherence Project study in Spain estimated a $75 \%$ adherence rate in patients with RRMS, using a very strict definition of adherence $(100 \%) .{ }^{13}$ However, the retrospective study by López-Méndez et $a l^{20}$ and the prospective study by McKay et $a l,{ }^{8}$ considering that a patient was adherent if the percentage of days covered by the medication dispensed was $\geq 80 \%$ and using different calculation systems, obtained adherence rates of $83.6 \%$ and $77.9 \%$, respectively, similar to our outcomes. There are other methods not considered in our study to measure adherence with lower feasibility, such as direct patient observation, questionnaires, electronic administration devices, remaining medication count or patient diaries.

We obtained higher adherence rates with GA, which were not statistically different from IFN $\beta 1 \mathrm{a}$-im. The risk of non-adherence with IFN $\beta 1 \mathrm{~b}$-sc is 3.5 times higher than with GA, and the main cause for this could be the adverse effects associated with interferons (although, according to our data, their severity is not sufficient to become the main cause for treatment interruption). However, the objective of this study was not to analyse the causes for lack of treatment adherence. The frequency of administration did not appear to be a decisive factor for adherence to parenteral DMTs. When comparing our outcomes with the published literature we found contradictory information. There are studies demonstrating that IFN $\beta 1 \mathrm{a}$-im is the drug with the highest rate of adherence ${ }^{7162122}$ while, on the other hand, the prospective studies by Santolaya $e t a l^{17}$ and McKay et $a l^{9}$ are in agreement with our results.

We have not detected significant differences for adherence in terms of gender, age or EDSS; however, there is a certain trend for higher adherence among women and young patients. There is no indication in our outcomes of any relationship between adherence and persistence, while the study on interferons by Vicente Iturbe $\mathrm{C}$ et $a l^{11}$ showed a higher discontinuation rate among non-adherent patients.

The limitations identified for this study are its retrospective and single-centre design and the limited size of the series analysed. The results must therefore be interpreted according to these limitations. In addition, for the adherence calculation we have assumed that all doses dispensed have been administered, which could lead to an overestimation of our results. Questionnaries should be included in the daily routine as a complement to confirm that the drug has been administered.

In summary, when comparing our results with other similar studies, we obtained lower persistence times, without identifying the causes, and similar discontinuation rates. EDSS was identified as an independent factor predicting treatment interruption. Regarding adherence, we have obtained results comparable to other studies, and it has been influenced by the drug used. However, we cannot claim that the differences found in adherence and persistence between the four treatment arms can be assigned only to those factors identified due to the limitations of our study and because there is a likely impact on adherence and persistence by other variables such as socioeconomic level, patient/healthcare staff relationship, disease denial, perception of lack of treatment benefit and adverse effects, motivation or family/social support. These variables showing the patient's point of view have been collected by authors such as Fox et $a l^{18}$ and Twork et $a l^{23}$ and we have not included them in our study.

Currently, oral drugs are being introduced into clinical practice, although parenteral DMTs continue to be prevalent. There is uncertain knowledge about the performance of these drugs beyond 2 years and there is a lack of comparative studies, which is why observational studies with prolonged follow-up times are relevant so that these drugs can be analysed comparatively. It is important to identify non-adherent patients, to analyse the reasons for this, and to develop individualised strategies in order to improve treatment adherence and efficacy; consequently, there will probably be an impact on persistence. Therefore, real-practice studies with adequate size and design are needed to collect clinical data on disease evolution and treatment efficacy, strict records that allow more accurate calculation of adherence rates and data from patients regarding health outcomes, which are particularly valuable in MS due to the characteristics of the treatments and disease. It is also necessary to reach consensus about calculation systems and definitions. Only then will we be able to identify and interpret accurately those factors that have an impact on persistence and adherence, which is information required in order to direct healthcare staff in their actions targeted to improving results in MS treatment.

\section{Key messages}

What is already known on this subject

- Multiple sclerosis is a chronic disease. This makes it relevant to study adherence and persistence due to the correlation between chronic conditions and the reduction in their rates.

- Patients with multiple sclerosis with higher adherence and persistence have a lower risk of relapses and a better quality of life, being associated with a lower use of healthcare resources.

- Multiple factors can have an influence on adherence and persistence, such as gender, age, EDSS, perceived loss of efficacy, adverse effects, problems with the injection device or dosing frequency.

What this study adds

- Additional data on persistence and adherence with DMTs.

- Although no significant differences in terms of persistence were found between drugs, patients on IFN $\beta 1$ a-im had a higher persistence rate.

- GA had the highest adherence rate, without major differences from IFN $\beta 1$ a-im.

- More evidence is provided about the factors that can influence both adherence and persistence, with EDSS being an independent predictor of treatment interruption according to the results.

Acknowledgements Elia Pérez Fernández (Research Unit at Hospital Universitario Fundación Alcorcón) for her assistance with statistical analysis.

Competing interests None declared.

Provenance and peer review Not commissioned; externally peer reviewed.

(C) European Association of Hospital Pharmacists (unless otherwise stated in the text of the article) 2019. All rights reserved. No commercial use is permitted unless otherwise expressly granted. 


\section{REFERENCES}

1 Ministerio de Sanidad, Servicios sociales e Igualdad. Estrategia en Enfermedades Neurodegenerativas del Sistema Nacional de Salud 2016 http://www.msps.es/ organizacion/sns/planCalidadSNS/pdf/Est_Neurodegenerativas_APROBADA_C_ INTERTERRITORIAL.pdf (accessed Oct 2016).

2 Lublin FD, Reingold SC, Cohen JA, et al. Defining the clinical course of multiple sclerosis: the 2013 revisions. Neurology 2014;83:278-86.

3 García Merino A, Fernández 0, Montalbán X, et al. Documento de consenso de la Sociedad española de Neurología sobre el uso de medicamentos en esclerosis múltiple: escalado terapéutico. Neurología 2010;25:378-90.

4 Sociedad Española de Farmacia Hospitalaria. Modelo de selección y atención farmacéutica de pacientes crónicos 2013 https://www.sefh.es/bibliotecavirtual/ Cronicos/AF_INFORME_PACIENTE_GESTION_SANITARIA.pdf (accessed Oct 2016).

5 Cramer JA, Roy A, Burrell A, et al. Medication compliance and persistence: terminology and definitions. Value Health 2008;11:44-7.

6 World Health Organization. Adherence to long-term therapies: evidence for action. 2003 http://www.who.int/chp/knowledge/publications/adherence_full_report.pdf? $\mathrm{ua}=1$ (accessed Jan 2016).

7 Halpern R, Agarwal S, Dembek C, et al. Comparison of adherence and persistence among multiple sclerosis patients treated with disease-modifying therapies: a retrospective administrative claims analysis. Patient Prefer Adherence 2011;5:73-84.

8 Costello K, Kennedy P, Scanzillo J. Recognizing nonadherence in patients with multiple sclerosis and maintaining treatment adherence in the long term. Medscape J Med 2008; 10:225

9 McKay KA, Tremlett H, Patten SB, et al. Determinants of non-adherence to diseasemodifying therapies in multiple sclerosis: a cross-Canada prospective study. Mult Scler 2017;23:588-96.

10 Correia I, Marques IB, Sousa M, et al. Predictors of first-line treatment persistence in a Portuguese cohort of relapsing-remitting multiple sclerosis. J Clin Neurosci 2016;36:73-8.

11 Vicente Iturbe C, Ara Callizo JR, Huarte Lacunza R, et al. [Discontinuation and longterm adherence to beta interferon therapy in patients with multiple sclerosis]. Farm Hosp 2012;36:77-83.
12 Steinberg SC, Faris RJ, Chang CF, et al. Impact of adherence to interferons in the treatment of multiple sclerosis: a non-experimental, retrospective, cohort study. Clin Drug Investig 2010;30:89-100.

13 Devonshire V, Lapierre Y, Macdonell R, et al. The Global Adherence Project (GAP): a multicenter observational study on adherence to disease-modifying therapies in patients with relapsing-remitting multiple sclerosis. Eur J Neurol 2011;18:69-77.

14 Andrade SE, Kahler KH, Frech F, et al. Methods for evaluation of medication adherence and persistence using automated databases. Pharmacoepidemiol Drug Saf 2006; 15:565-74

15 Evans C, Marrie RA, Zhu F, et al. Adherence and persistence to drug therapies for multiple sclerosis: a population-based study. Mult Scler Relat Disord 2016;8:78-85.

16 Reynolds MW, Stephen R, Seaman C, et al. Persistence and adherence to disease modifying drugs among patients with multiple sclerosis. Curr Med Res Opin 2010;26:663-74.

17 Santolaya R, Fernández-Pacheco M, Arteche L, et al. Adherencia subóptima al tratamiento en la esclerosis multiple. Adherence to treatment in multiple sclerosis. Farm Hosp 2012;36:124-9.

18 Fox RJ, Salter AR, Tyry T, et al. Treatment discontinuation and disease progression with injectable disease-modifying therapies: findings from the North American Research Committee on Multiple Sclerosis database. Int J MS Care 2013;15:194-201.

19 Kripalani S, Yao X, Haynes RB. Interventions to enhance medication adherence in chronic medical conditions: a systematic review. Arch Intern Med 2007;167:540-50.

20 López-Méndez P, Río J, Pérez-Ricart A, et al. [Therapy adherence to immunomodulator treatment in patients with multiple sclerosis]. Rev Neurol 2013;56:8-12.

21 Hansen K, Schüssel K, Kieble M, et al. Adherence to disease modifying drugs among patients with multiple sclerosis in Germany: a retrospective cohort study. PLoS One 2015; 10:e0133279.

22 Kleinman NL, Beren IA, Rajagopalan K, et al. Medication adherence with disease modifying treatments for multiple sclerosis among US employees. J Med Econ 2010;13:633-40.

23 Twork S, Nippert I, Scherer P, et al. Immunomodulating drugs in multiple sclerosis: compliance, satisfaction and adverse effects evaluation in a German multiple sclerosis population. Curr Med Res Opin 2007;23:1209-15. 